\title{
Effects of average grain size on the magnetic properties of permalloy films
}

\author{
Nikolay Djuzhev ${ }^{1}$, Aleksey Iurov $^{1}$, Nikita Mazurkin ${ }^{1}$, Maksim Chinenkov $^{1, *}$, Aleksey Trifonov ${ }^{2}$ and Marina Pushkina ${ }^{1}$ \\ ${ }^{1}$ National Research University of Electronic Technology (MIET), 124498, Moscow, Russia \\ ${ }^{2}$ F.V. Lukin State Scientific-Reseach Institute of Physical Problems, 124460, Moscow, Russia
}

\begin{abstract}
In this paper the results of substrate temperature effects on average grain size in FeNi 20:80 are shown. It was found that with an increase of the substrate temperature from 270 to $390{ }^{\circ} \mathrm{C}$, the anisotropic magnetoresistive (AMR) effect increases from 1.2 to $2.3 \%$ and the coercive force from 1.6 to 5.3 Oe. The presence of two conflicting processes: the task of increasing AMR effect to achieve high sensitivity of AMR-sensor and to decrease the coercive force to increase the precision of conversion, leads to the necessity to find the optimum deposition temperature of the permalloy film. The dependence of the AMR effect and the coercive force on the substrate temperature during deposition was obtained. This dependence shows that the substrate temperature increasing above $320^{\circ} \mathrm{C}$ leads to coercive force increasing and does not lead to a substantial AMR increasing. In this regard, the substrate temperature $320^{\circ} \mathrm{C}$ was determined as optimal.
\end{abstract}

\section{Introduction}

The ability of the magnetic thin films to change its resistance under the influence of an external magnetic field is applied in the creation of effective magnetic field measurement devices [1, 2, 3, 4, 5]. Permalloy films FeNi widely used to create anisotropic magnetoresistive sensors (AMR sensors), which convert magnetic field into an electrical signal due to a change in conductivity with a change of the angle between the current vector and the magnetization vector in the permalloy film. The AMR effect is expressed by the formula:

$$
\rho(\theta)=\rho_{0}+\Delta \rho \cos ^{2} \theta
$$

where $\theta$ - the angle between the magnetization vector and the electrical current direction, $\Delta \rho / \rho_{0}-$ the magnetoresistivity coefficient.

To ensure a high level of output characteristics of AMR sensors it is needed to improve the magnetic properties of the magnetoresistive material. In particular it is needed to maximize the AMR effect, which determines the output signal amplitude, and to prevent the strong growth of the coercive force and anisotropy field for maximum sensitivity and minimum hysteresis achievement. However, it should be taken into account that the magnetoresistive material parameters are determined not only by its internal characteristics, but significantly by the composition and parameters of the film growth, particularly average grain size.

\section{Experiment}

Permalloy films were obtained by magnetron deposition on a Phase II AJA unit. The target diameter is
$50 \mathrm{~mm}$, the distance to the substrate is $112 \mathrm{~mm}$, the thickness of the working and dielectric layers is given in Table 1.

Table 1. Test structure.

\begin{tabular}{|l|l|}
\hline \multicolumn{1}{|c|}{ Layer } & \multicolumn{1}{c|}{$\begin{array}{c}\text { Estimated layer } \\
\text { thickness, } \mathrm{nm}\end{array}$} \\
\hline $\mathrm{FeNi} 20: 80$ & 30 \\
\hline $\mathrm{Si}_{3} \mathrm{~N}_{4}$ & 120 \\
\hline $\mathrm{SiO}_{2}$ & 600 \\
\hline $\mathrm{Si}$ & substrate \\
\hline
\end{tabular}

To create the anisotropy and the AMR in the magnetron deposition camera magnetic field was generated at least 40 Oe.

In this article an experiment was conducted to determine the impact of process parameters on the deposition rate of the material and the grain size in films of FeNi 20:80 during magnetron deposition. To establish the relationship between the characteristics of the films of permalloy and output parameters AMR sensors, primarily sensitivity a complex of studies showing a significant influence of the substrate temperature on the average grain size in the film of permalloy (fig. 1) at a constant magnetron power of $150 \mathrm{~W}$ and a working gas pressure of $0.5 \mathrm{~Pa}$ was conducted. The average grain size measurement was conducted on the multifunctional $\mathrm{x}$ ray analytical systems "RIKOR-8", "X-Ray MiniLab-6".

Corresponding author: chinenkov@,inbox.ru 


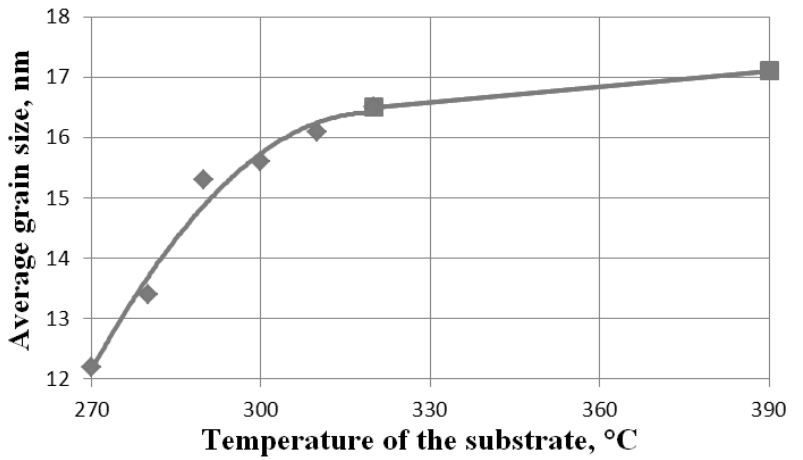

Fig. 1. The dependence of the average grain size on the substrate temperature in the FeNi film.

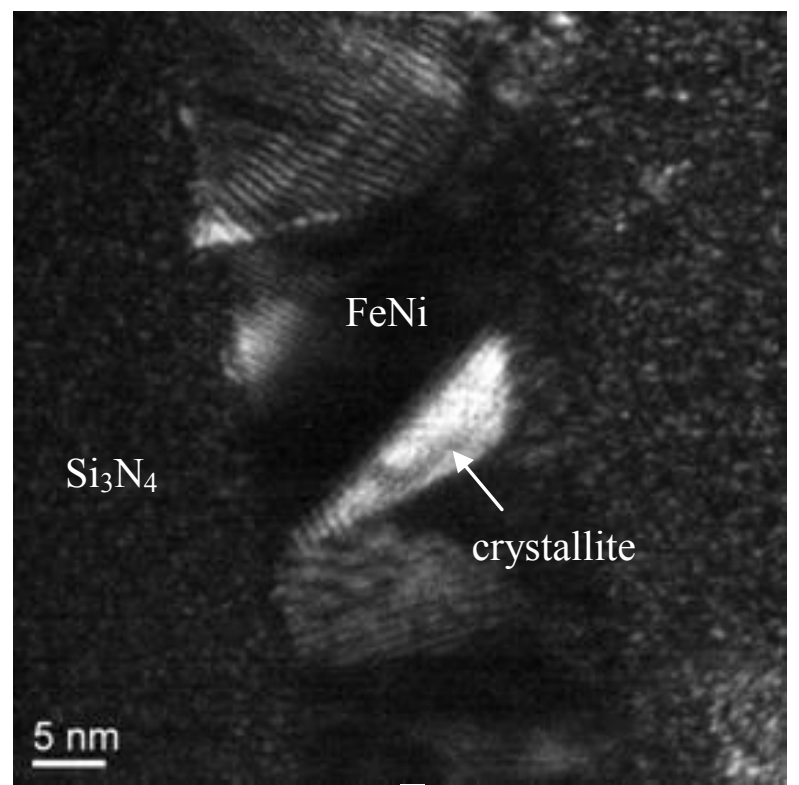

a)

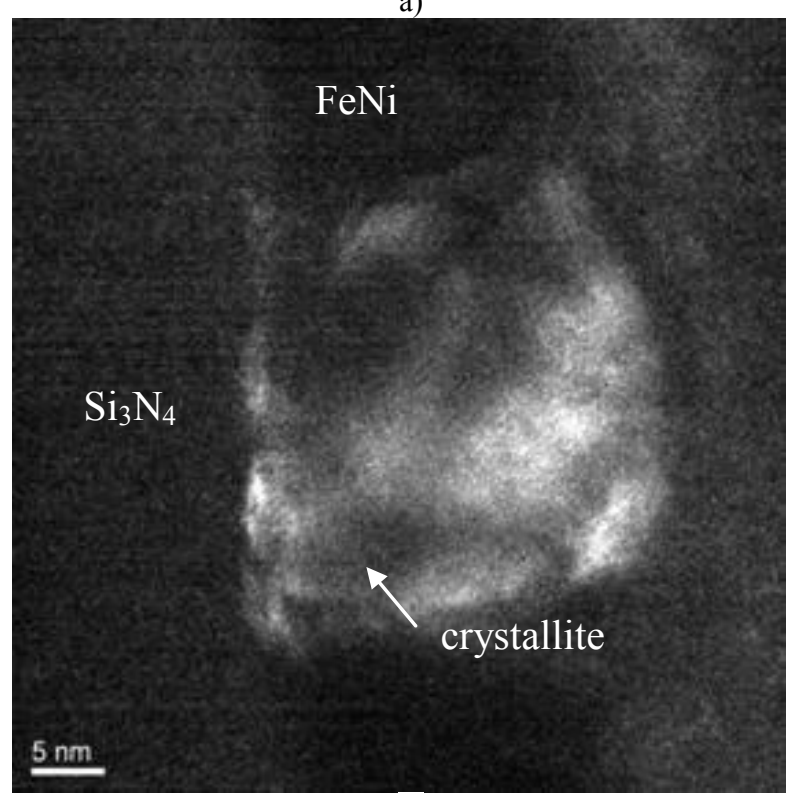

b)

Fig. 2. The image of the grain in the dark field obtained on a transmission electron microscope. a) the substrate temperature during the deposition was $270{ }^{\circ} \mathrm{C}$; b) the substrate temperature during the deposition was $390{ }^{\circ} \mathrm{C}$.
Figure 2 shows photographs taken on TEM grown at different substrate temperatures. It can be seen from the figure that when the substrate temperature increases from 270 a) to $390{ }^{\circ} \mathrm{C}$, the size of crystallite and the surface roughness increases. The increase in the surface roughness is confirmed by atomic force microscope, Fig. 3 . It can be seen from the figure that the average height of particles increased from 0.75 to $5.85 \mathrm{~nm}$ (on the sidebar to the figures).Figure 1 shows that average grain size increases significantly to a temperature of $300-320$ ${ }^{\circ} \mathrm{C}$. Above this temperature grain growth is slowing sharply. We assume that this transition is associated with the achievement of the average grain size of the film thickness and further growth leads to the development of the surface topography, as evidenced by research on transmission electron microscope and atomic force microscope, fig. 2 and fig. 3 .
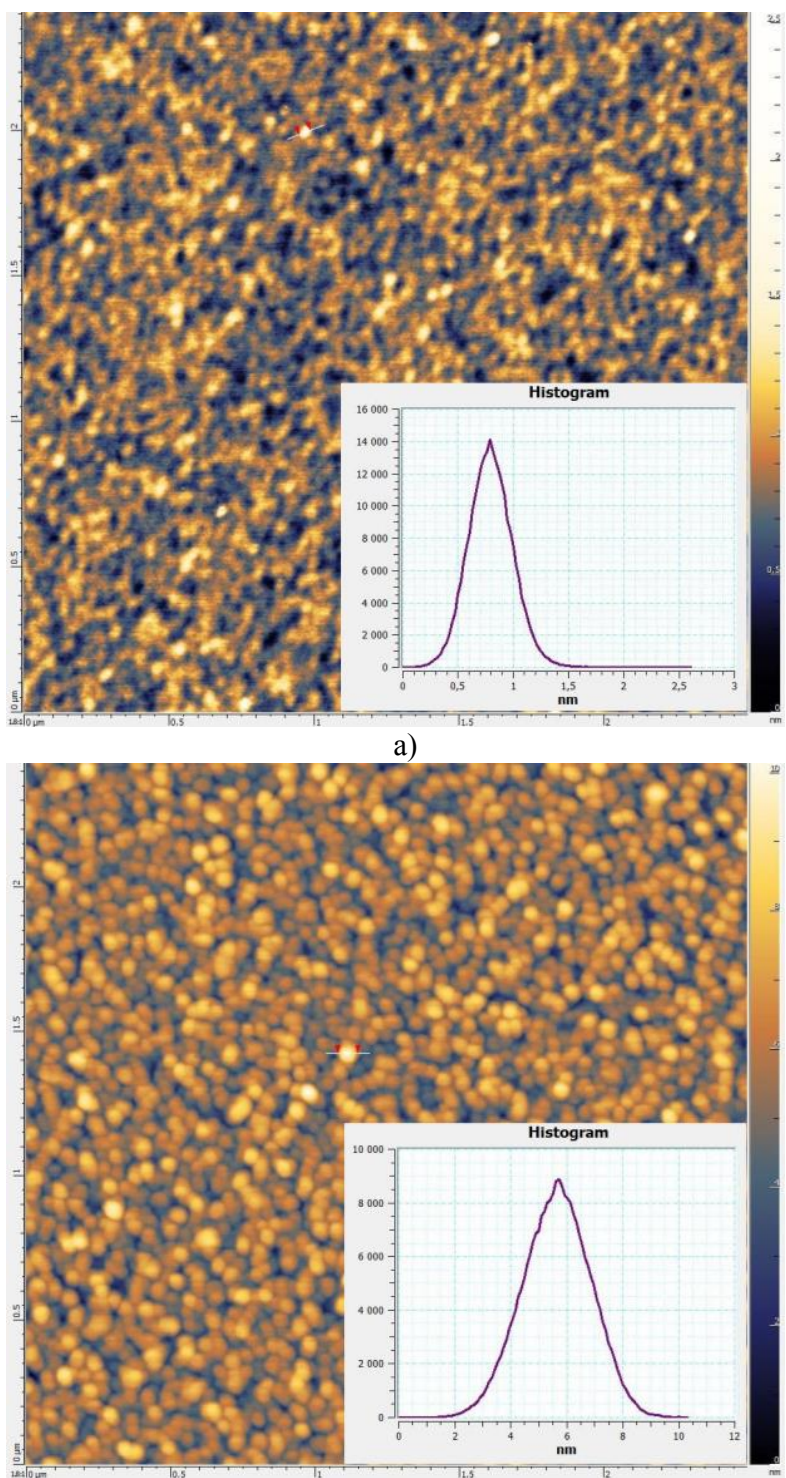

b)

Fig. 3. The surface image of the permalloy films at different deposition temperatures taken with AFM AIST-NT Smart SPM-1000. a) the substrate temperature during the deposition was $270{ }^{\circ} \mathrm{C}$, Ra is equal to $0.16 \mathrm{~nm}$, and b) the substrate temperature during the deposition was $390{ }^{\circ} \mathrm{C}$, Ra is equal to $0.97 \mathrm{~nm}$. 
It is shown that grain size increasing lead to increase of the magnitude of the AMR and the coercivity (fig.4). The presence of two conflicting processes, namely the AMR increasing for high sensitivity achievement and a coercivity decreasing to increase the precision of conversion, leads to the necessity of finding the optimum deposition temperature of the permalloy film.To determine the optimal deposition temperature based on the data presented in fig. 1 and fig. 4, the dependence of the AMR effect and the coercive force on the substrate temperature during the deposition was obtained (fig. 5). This dependence shows that the substrate temperature increasing above $320{ }^{\circ} \mathrm{C}$ leads to coercivity increasing and does not lead to a substantial AMR increasing. In this regard, the temperature $320{ }^{\circ} \mathrm{C}$ was determined as optimal.

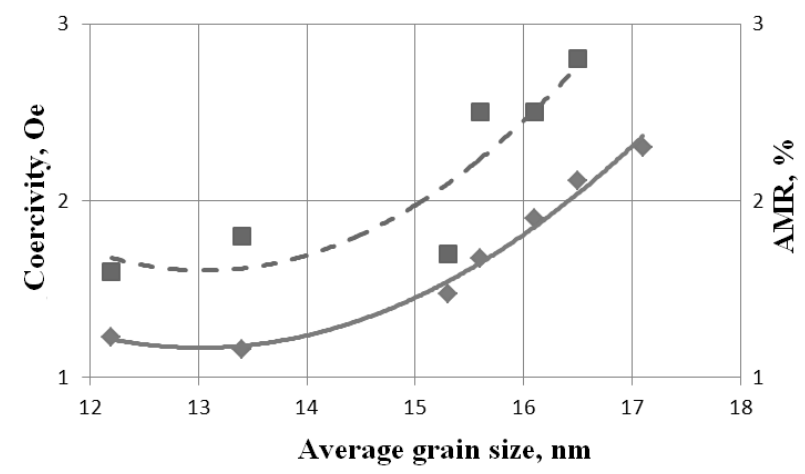

Fig. 4. The dependence of the coercivity (dashed line) and the AMR (solid line) on the average grain size.

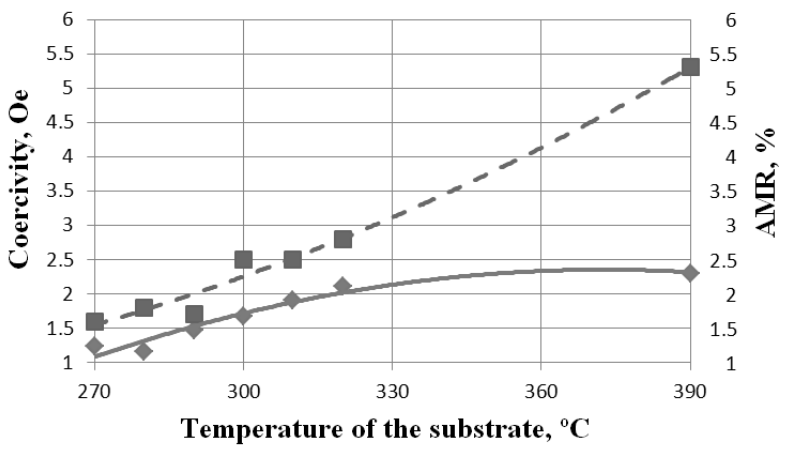

Fig. 5. The dependence of the coercivity (dashed line) and the AMR (solid line) on the substrate temperature.

Other magnetron deposition parameters affect the average grain size and output signal of AMR sensors much weaker. The results of the experimental study parameters of the permalloy film from the substrate temperature are given in table 2 . The diffraction patterns were measured in a standard symmetrical scanning scheme with Bragg-Brentano focusing, Fig. 6. The size distribution of the crystallites is approximated by the log-normal distribution, estimated by the WarrenAverbach method.

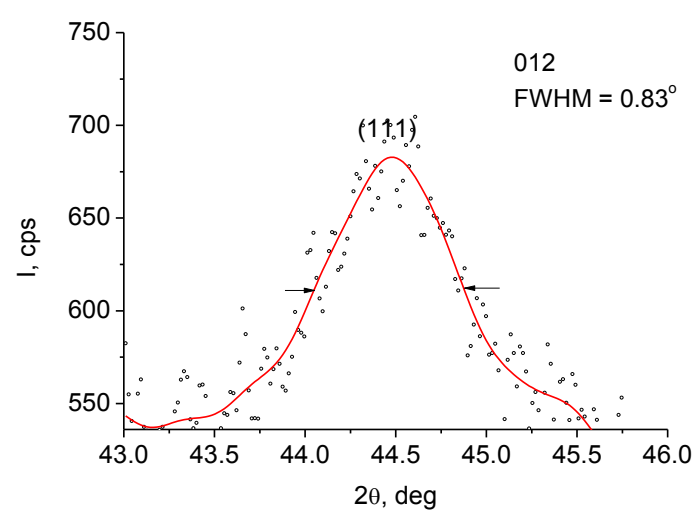

a)

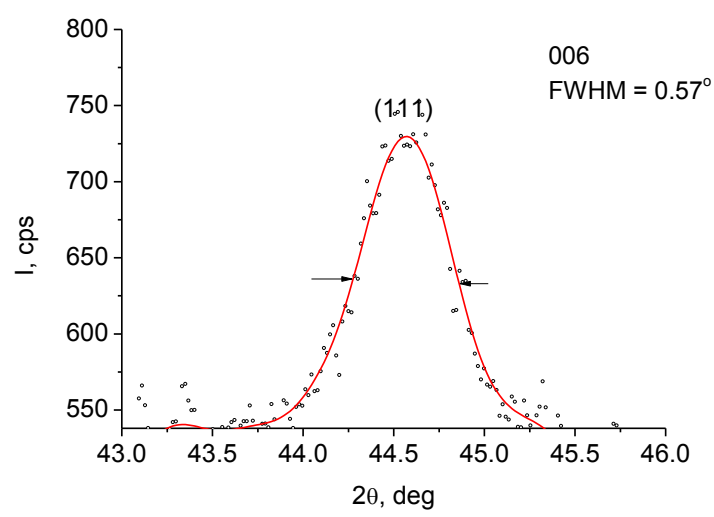

б)

Fig. 6. The diffraction peak of permalloy film for the substrate temperature: a) $270{ }^{\circ} \mathrm{C}$; b) $390{ }^{\circ} \mathrm{C}$.

It is known that the AMR increases with the permalloy film thickness increasing. However, with the thickness increasing the conductivity of the layer grows, and the resistance of the diagonal of the bridge AMR sensor decreases, which leads to significant heating and temperature characteristics deterioration. Therefore, the magnetoresistive layer thickness is selected maximum on the basis of the requirements of achieving the resistance of the bridge diagonal, the values of $1.5 \mathrm{kOhm}$ in an existing topology (fig.7).

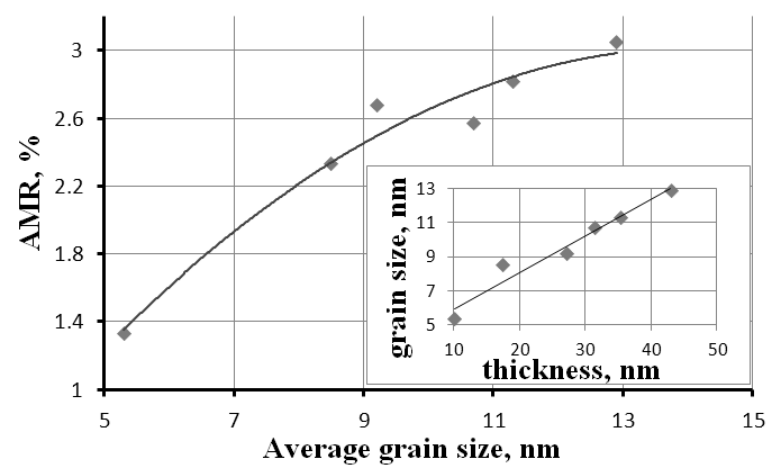

Fig. 7. The dependence of the AMR on the average grain size and film thickness. 
Table 2. The dependence of the parameters of the FeNi 20:80 permalloy film on the substrate temperature.

\begin{tabular}{|c|c|c|c|c|c|c|}
\hline $\begin{array}{c}\text { Substrate } \\
\text { temperature, } \\
{ }^{\circ} \mathrm{C}\end{array}$ & $\begin{array}{c}\text { AMR, } \\
\%\end{array}$ & $\begin{array}{c}\text { Coercivity, } \\
\text { Oe }\end{array}$ & $\begin{array}{c}\text { Anisotropy, } \\
\text { Oe }\end{array}$ & $\begin{array}{c}\text { Average grain } \\
\text { size, } \\
\text { nm }\end{array}$ & $\begin{array}{c}\text { film } \\
\text { thickness, } \\
\text { nm }\end{array}$ & $\begin{array}{c}\text { Surface } \\
\text { resistance, } \\
\text { Ohm }\end{array}$ \\
\hline 390 & 2.30 & 5.3 & 7 & 17.1 & 26.6 & 8.25 \\
\hline 320 & 2.01 & 2.8 & 6 & 16.5 & 26.6 & 8.21 \\
\hline 310 & 1.90 & 2.5 & 5 & 16.1 & 26.5 & 8.48 \\
\hline 300 & 1.82 & 2.5 & 6 & 15.6 & 25.5 & 8.92 \\
\hline 290 & 1.47 & 1.7 & 6 & 15.3 & 26.6 & 8.57 \\
\hline 280 & 1.16 & 1.8 & 7 & 13.4 & 26.7 & 8.88 \\
\hline 270 & 1.23 & 1.6 & 7 & 12.2 & 26.7 & 9.29 \\
\hline
\end{tabular}

\section{Conclusion}

Based on the results of experiments the substrate temperature $320{ }^{\circ} \mathrm{C}$ was determined as optimal for the installation of Phase II AJA 150. The magnetic field sensor based on the investigated permalloy films were made. This sensor had a sensitivity value 9.4 $(\mathrm{mV} / \mathrm{V}) /(\mathrm{kA} / \mathrm{m})$ in the bias field value of $1 \mathrm{kA} / \mathrm{m}$ instead of $3.0(\mathrm{mV} / \mathrm{V}) /(\mathrm{kA} / \mathrm{m})$.

The work was supported by the Minobrnauki RF (Goszadanie № 16.2475.2017/4.6) and conducted using the equipment of Multi-access center «Microsystem technics and electronic component base» MIET.

\section{References}

1. S. Tumanski, Thin Film Magnetoresistive Sensors (IOP Publishing Ltd, 2001).

2. Dibbern, U. Magnetoresistive Sensors, in: W.Gopel, J. Hesse, J. N. Zemel, Sensors vol. 5, Copyright VCH Verlagsgesellschaft mbH, Hannover, 1989, pp. $341-380$.

3. N.A. Djuzhev, N.S. Mazurkin, V.S. Pozdnyakov, A.S. Iurov, M.Yu. Chinenkov. Semiconductors, Vol. 49, № 13, pp. 1739-1742 (2015).

4. V. Bespalov, N. Djuzhev, A. Iurov, N. Mazurkin, M. Chinenkov, R. Preobrazhensky, Solid State Phenomena, 249 (2016).

5. V. Bespalov, N. Djuzhev, A. Iurov, N. Mazurkin, M. Chinenkov, R. Preobrazhensky Journal of Surface Investigation: X-ray, Synchrotron and Neutron Techniques, Vol. 11, No. 2, pp. 343-345 (2017). 